\title{
Modelling and analysis of metal transfer in gas metal arc welding
}

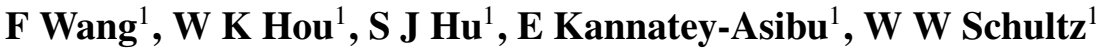 \\ and P C Wang ${ }^{2}$ \\ ${ }^{1}$ Department of Mechanical Engineering, University of Michigan, Ann Arbor, \\ Michigan, USA \\ ${ }^{2}$ General Motors R\&D and Planning Center, Warren, Michigan, USA
}

Received 8 October 2002, in final form 12 March 2003

Published 16 April 2003

Online at stacks.iop.org/JPhysD/36/1143

\begin{abstract}
A numerical model combining the methods of enthalpy, effective-viscosity and volume-of-fluid is developed to simulate the metal transfer process in gas metal arc welding. The model describes not only the influence on droplet profile and transfer frequency of electromagnetic force, surface tension, and gravity, but it can also model the nonisothermal phenomena such as heat transfer and phase change. The model has been used to study the shape of the melting interface on the welding wire, the droplet oscillation at wire tip, the characteristics of relevant physical variables and their roles in metal transfer. We find that the taper formation in spray transfer is closely related to the heat input on the unmelted portion of the welding wire, and the taper formation affects the globular-spray transition by decelerating the transfer process. The formation of satellite drops during the metal transfer process is also considered. High-speed photography, laser-shadow imaging, and metallographic analysis validate the numerical model, and recommendations are made on the topics that require further consideration for a more accurate metal transfer model.
\end{abstract}

\section{Introduction}

Metal transfer in gas metal arc welding (GMAW) refers to the process of transferring material of the welding wire in the form of molten liquid droplets to the workpiece. Metal transfer plays an important role in process stability and weld quality. Depending on the welding conditions, metal transfer can take place in three principal modes: globular, spray, and short-circuiting. Globular transfer, where the droplet diameter is larger than the wire diameter, occurs at low current. Since it is often accompanied by excessive spatter, globular transfer is only used in welding unimportant parts. Spray transfer, where the droplet diameter is smaller than the wire diameter, occurs at medium and high current. It is a highly stable and efficient process, and is widely used in welding thick steel plates and aluminium parts. Short-circuiting transfer is a special transfer mode where the molten droplet on the wire tip makes direct contact with the workpiece or the surface of the weld pool. It is characterized by repeated, intermittent arc extinguishment and re-ignition. It requires low heat input and hence is commonly used in welding thin sheets.

Due to the wide use of GMAW in industry, numerous models have been developed to study the metal transfer process
[1-14]. Typical approaches include the static force balance theory $[2,3]$, the pinch instability theory $[4,5]$, the marker-andcell method [6], the volume-of-fluid (VOF) method [12, 13], and one-dimensional analysis [10,14]. By considering the effects of surface tension, gravity and electromagnetic force, these models were able to predict the metal transfer frequency or the droplet shape in one or more transfer modes. However, heat transfer and phase change effects were ignored in these models, and the object considered was typically an alreadymelted liquid droplet hanging on a solid wire that has a flat end. In fact, as will be shown in this paper, the melting interface is neither flat nor steady, and the heat transfer and phase change effects have significant impact on the metal transfer modes. As a result, these models cannot adequately describe the metal transfer process nor predict the geometry of the melting interface, the self-regulation of wire extension, and the taper formation at higher current.

In 1996, Haidar and Lowke [15] developed a nonisothermal model based on the VOF method to simulate the metal transfer in globular and spray modes. This was the first time that the VOF method was used in metal transfer simulation and it made a great impact on this field. 
The energy equation, as well as continuity, momentum and current equations, was solved in both the molten droplet and surrounding plasma arc, and the shape of the droplet was predicted as a function of time. However, later high-speed observations showed that the droplet shapes they predicted were not accurate enough and the 'mixture of small and large drops' at the current of globular-spray transition was not observed. In 1998, Fan and Kovacevic [16,17] developed a more comprehensive nonisothermal model to simulate the droplet formation, detachment and impingement on the weld pool. Their results were compared and agreed well with experiments with a high-speed video camera. Unfortunately, only globular transfer was considered in their two papers. In 2001, Wang and Tsai [18] used another nonisothermal model to simulate the droplet impingement on the weld pool surface and the consequent fluid flow in the weld pool. While their paper focused on the interaction between the droplet and the weld pool, the mechanism of droplet detachment was not studied.

In this paper, we combine the enthalpy, effective-viscosity and VOF methods to develop a more general numerical model to simulate the metal transfer process. The new model not only describes the dynamic droplet shape and detachment frequency, but it also predicts the geometry of the melting interface and the nonisothermal phenomena during the process such as heat transfer and phase change. With the model, the physics of the GMAW process, especially the mechanisms of droplet detachment, taper formation and globular-spray transition, can be studied in depth. The generality of the algorithms in handling heat transfer, phase change, fluid flow, surface deformation and electromagnetic forces allow it to be extended to a complete GMAW model, simulating the metal transfer and weld pool dynamics simultaneously. The complete GMAW model will provide a mathematical platform for further study on welding physics, the characteristics of microstructure, and the system response in closed-loop control. Due to the length limitation of this paper and the unique characteristics associated with short-circuiting transfer, only globular and spray modes will be discussed.

\section{Mathematical formulation and numerical procedure}

A two-dimensional axisymmetric coordinate system is used to model the metal transfer in GMAW, as shown in figure 1, where the dashed box indicates the computational domain. The inner boundary of the domain is located on the symmetry axis; the top boundary is attached to the bottom surface of the contact tube and passes through the welding wire; the outer and bottom boundaries are located to include the complete wire extension and molten droplets in the computational domain. A detached droplet is initially included in the computation, but erased when it approaches the bottom boundary. This will simplify the algorithm as we focus our attention on the physics of droplet formation and detachment.

In addition to the symmetry condition, the following assumptions have been made:

(a) The liquid metal is an incompressible Newtonian fluid.

(b) Material properties in the same phase are constants.

(c) Chemical reaction and metal vaporization are negligible.

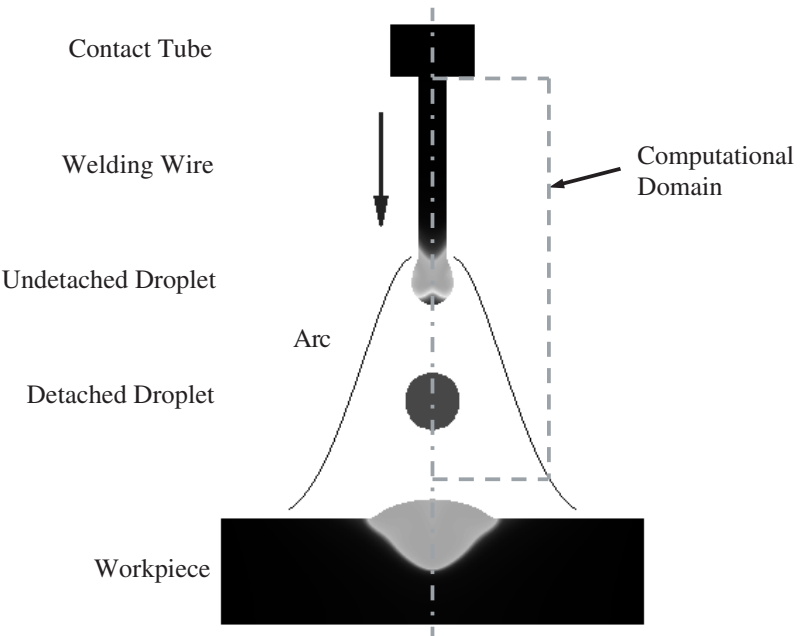

Figure 1. Schematic of the metal transfer process in GMAW.

(d) Boundary conditions of heat flux and current density are prescribed as shown later.

Among these assumptions, some factors, e.g. the metal vaporization and the assumed heat flux boundary condition, may affect the metal transfer by a greater degree. Recommendations for future study on these factors will be made after analysing the results with the assumptions.

\subsection{Principal methods and governing equations}

Inside the computational domain are three phases: a solid phase (the unmelted wire), a liquid phase (the molten droplets), and a gas phase (the plasma and shielding gas). By combining the enthalpy and the effective-viscosity methods [19], however, the solid and liquid phases can be considered as one phase: a generalized liquid phase where the solid region has an extremely high viscosity $\left(10^{8}\right.$ times the viscosity of the liquid metal). With this method, the location and geometry of the solid-liquid interface can be automatically determined, and no boundary conditions are necessary on this interface. This technique was first used by Kou and Sun [19] and adopted by other authors in their weld pool models. It proved to be efficient and accurate.

The generalized liquid phase is surrounded partially by the free surface of the molten liquid. To solve the differential equations in the generalized liquid phase, the motion of the free surface needs to be tracked so that boundary conditions can be properly applied. This is done in the present model by using the VOF method [20]. This method was developed at Los Alamos National Laboratory in 1980 to study the transient flow with free surfaces. Because of its numerical stability and efficiency, the VOF method has been applied in many industrial fields, such as inkjet printing, spray plating, welding, etc. A problem of the VOF method is that, sometimes, nonphysical voids or fluid filaments are generated and propagate throughout the computational domain. However, with proper book-keeping or surface smoothing, these voids and filaments can be suppressed and good simulation of the free surface can be achieved.

Based on these methods and assumptions, the following governing equations are derived for the generalized 
liquid phase:

Mass continuity equation

$$
\nabla \cdot \vec{v}=0
$$

Momentum equation

$$
\frac{\partial \vec{v}}{\partial t}+\vec{v} \cdot \nabla \vec{v}=-\frac{1}{\rho} \nabla P+\nu_{\mathrm{e}} \nabla^{2} \vec{v}-\vec{J} \times \vec{B}+\vec{g}
$$

Energy equation $\quad \frac{\partial h}{\partial t}+\vec{v} \cdot \nabla h=k \nabla^{2} T+\frac{\vec{J} \cdot \vec{J}}{\sigma}$

Current continuity equation $\quad \nabla \cdot(\sigma \nabla V)=0$

Maxwell's equation $\quad \oint \vec{B} \cdot \mathrm{d} \vec{s}=\mu_{0} I$

Ohm's law $\quad \vec{J}=-\sigma \nabla V$

Enthalpy-temperature relation

$$
T= \begin{cases}T_{\mathrm{m}}+\frac{h}{c_{\mathrm{s}}} & (H \leqslant 0) \\ T_{\mathrm{m}} & (0<H<L) \\ T_{\mathrm{m}}+\frac{h-L}{c_{1}} & (H \geqslant L)\end{cases}
$$

$$
\text { Effective-viscosity } \quad v_{\mathrm{e}}= \begin{cases}v & \left(T \geqslant T_{\mathrm{m}}\right) \\ 10^{8} \times v & \left(T<T_{\mathrm{m}}\right)\end{cases}
$$

where $\vec{v}$ is the fluid velocity, $P$ is the hydrodynamic pressure, $\vec{J}$ is the current density, $\vec{B}$ is the magnetic field, $T$ is the temperature, $h$ is the enthalpy, $V$ is the electric potential (voltage), and $I$ is the welding current. Material properties include density $\rho$, effective-viscosity $v_{\mathrm{e}}$, kinematic viscosity $v$, thermal conductivity $k$, electrical conductivity $\sigma$, melting temperature $T_{\mathrm{m}}$, latent heat $L$, specific heat of solid $c_{\mathrm{s}}$, and specific heat of liquid $c_{1}$. The permeability of free space $\mu_{0}=4 \pi \times 10^{-7} \mathrm{H} \mathrm{m}^{-1}$ and the gravitational acceleration $g=9.8 \mathrm{~m} \mathrm{~s}^{-2}$.

\subsection{Boundary conditions}

There are two types of boundary conditions. One is on the boundary of the computational domain, and the other is on the free surface. Boundary conditions on the finite-difference domain are shown in figure 2. The top surface is a flow inlet, whose temperature and voltage are given. The symmetry axis is shown by the chain dashed line, where the radial gradients of physical variables are zero. As mentioned earlier, in globular and spray transfer, the detached droplets are erased when they approach the bottom of the computational domain. Therefore, no liquid can reach the bottom boundary, as well as the outer boundary. Consequently, no boundary conditions at these locations are necessary. In figure $2, v_{\text {feed }}$ is the wire feed speed (WFS), $R_{\text {wire }}$ is the radius of the welding wire, and $U_{\mathrm{w}}$ is the applied voltage.

The boundary conditions on the free surface are as follows:

(a) The normal stress is induced by surface tension:

$$
\vec{n} \cdot \underline{\underline{\tau}} \cdot \vec{n}=-\gamma\left(\frac{1}{R_{1}}+\frac{1}{R_{2}}\right)
$$

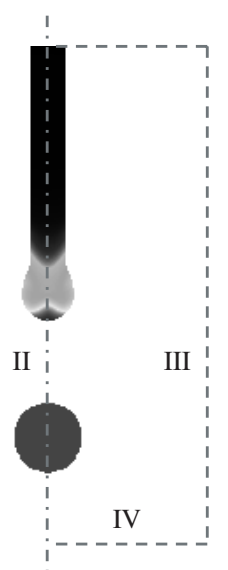

I: $\quad$ Flow inlet

$v_{z}=-v_{\text {feed }}\left(r \leq R_{\text {wire }}\right)$

$v_{r}=0, \quad \frac{\partial P}{\partial z}=0$,

$T=T_{\text {room }}, \quad V=U_{w}$

II: Symmetry axis $\quad v_{r}=0, \quad \frac{\partial v_{z}}{\partial r}=0$

$\frac{\partial P}{\partial r}=0$

$\frac{\partial T}{\partial r}=0, \quad \frac{\partial V}{\partial r}=0$.

No boundary conditions on III and IV are necessary. (See the text for explanation)

Figure 2. Boundary conditions of the finite-difference domain.

or

$$
-P+2 \mu \frac{\partial v_{\mathrm{n}}}{\partial n}=-\gamma\left(\frac{1}{R_{1}}+\frac{1}{R_{2}}\right)
$$

where $\underline{\underline{\tau}}$ is the stress tensor, $\vec{n}$ is the normal direction vector, $n$ is the scalar distance along $\vec{n}, \gamma$ is the surface tension coefficient, $\mu$ is the dynamic viscosity $(\mu=\rho \cdot v), v_{\mathrm{n}}$ is the velocity component in the normal direction, and $R_{1}$ and $R_{2}$ are the two principle radii of curvature.

(b) The shear stress is assumed to be zero:

$$
\vec{n} \cdot \underline{\underline{\tau}} \cdot \vec{t}=0 \quad \text { or } \quad \mu\left(\frac{\partial v_{\mathrm{n}}}{\partial t}+\frac{\partial v_{\mathrm{t}}}{\partial n}\right)=0
$$

where $\vec{t}$ is the tangential direction vector, $t$ is the scalar distance along $\vec{t}$, and $v_{\mathrm{t}}$ is the velocity component in the $t$-direction. The Marangoni effect (shear stress due to surface tension gradient) is ignored in this paper for two reasons:

1. The influence of the Marangoni effect on droplet detachment is much smaller than that of the Lorentz force. In the weld pool, the Marangoni and Lorentz effects are comparable (although Kou and Sun [19] find that the Lorentz force has greater influence on the weld pool convection). But due to the bell-shaped arc distribution, the current density on the droplet is much higher than that on the pool surface. We know that the pinch force (horizontal Lorentz force) is roughly proportional to the square of current density:

$$
F_{r}=-J_{z} B_{\theta}=-J_{z} \frac{\mu_{0}}{r} \int_{0}^{r} J_{z} r \mathrm{~d} r \approx-J_{z}^{2} \frac{\mu_{0} r}{2} .
$$

The Lorentz force is therefore enhanced on the droplet. No evidence shows that the Marangoni coefficient is strongly affected by current density.

2. According to Cobine and Burger [21] and Nemchinsky $[22,23]$, the highest temperature on droplet surface is near or above the boiling point and heavy vaporization occurs on the surface. Hence, measurement and modelling of the Marangoni effect are very difficult.

(c) The current density on the surface is approximated by a function linear in the axial component $z$, as proposed and tested by Choi et al [12]:

$$
J_{\mathrm{n}}(z)=\frac{I \cdot z}{\int z \cdot \mathrm{d} A}
$$


where $I$ is the welding current, $\mathrm{d} A$ is the local surface area, and $z$ is the vertical distance measured from the melting point on the wire surface to the location of $\mathrm{d} A$, as shown in figure $3(a)$. With this function, no current leaves the welding wire from locations above the melting point. Choi et al [12], found the predicted results with this function were in broad agreement with experiments.

(d) The heat flux around the molten droplet and the welding wire is complex. A simple way to approximate the heat flux distribution is to use a linear function similar to the current density distribution. However, that function will eliminate all heat transfer on the unmelted portion of the welding wire. Since the temperature of the arc can be as high as $20000 \mathrm{~K}$, the arc heats not only the molten droplet, but also the unmelted wire surface to an elevated temperature before it reaches the melting interface. The heat input on the welding wire was considered by many researchers [24-26] as an important factor in their metal transfer or heat transfer models. We find that, as will be shown later, neglecting the heat input on the unmelted wire will lead to poor modelling of the taper formation at high welding current and relatively large discrepancies in the predicted metal transfer frequency. To maintain the mathematical simplicity while including the heat flux on the unmelted wire, we keep a linear function to approximate the heat flux but consider that the extent of heat flux is somewhat larger to include a portion of the unmelted wire surface. In other words, we use a linear function whose starting point is located at a short distance $\Delta z$ above the melting location on the surface:

$$
q_{\mathrm{n}}\left(z^{\prime}\right)=\frac{\eta \eta_{\mathrm{d}} U I \cdot z^{\prime}}{\int z^{\prime} \cdot \mathrm{d} A}
$$

where $U$ is the arc voltage, $\eta$ is the arc efficiency or the ratio of the heat absorbed by molten metal to the heat generated by the arc, $\eta_{\mathrm{d}}$ is the ratio of the heat absorbed by the droplet to the total heat absorbed by the molten metal, and $z^{\prime}$ is the vertical distance measured from the origin, located at a short distance, $\Delta z$, above the melting point on the wire surface, or $z^{\prime}=z+\Delta z$, as shown in figure $3(b)$. The influence of the value of $\Delta z$ will be discussed in the next section, but for most of the computation in this paper, $\Delta z=R_{\text {wire }} / 2$ is selected since the discussion shows that $\Delta z=R_{\text {wire }} / 2$ gives good prediction of both melting interface geometry and metal transfer frequency.

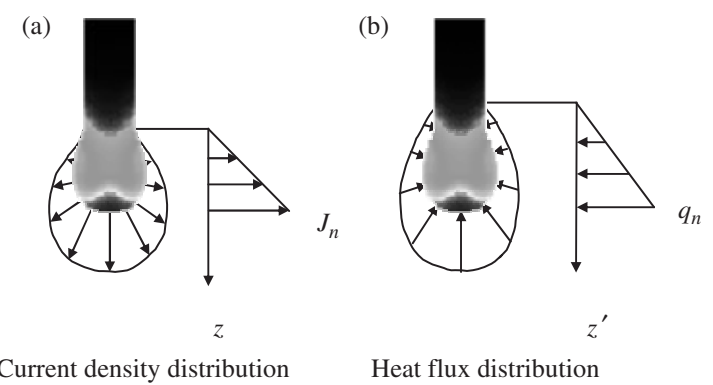

Figure 3. Boundary conditions of current density and heat flux on the free surface. (a) The origin of the current density distribution is located at the melting point on wire surface; and $(b)$ the origin of the heat flux distribution is located at a short distance, $\Delta z$, higher than the melting point so that the heat transfer on unmelted wire is included in calculation.
According to Grong [27], $\eta \approx 0.7$ in GMAW; and according to Wang and Tsai [18], $\eta_{\mathrm{d}} \approx 0.3$.

\subsection{Numerical procedure}

Numerical routines have been developed based on the discretized governing equations and boundary conditions. A uniform mesh with $36 \times 400$ square cells is used since nonuniform meshes do not provide particular benefits when they significantly increase the model complexity and computational time. The radius of the welding wire is covered by 12 cells, and the time increment is $5 \times 10^{-6} \mathrm{~s}$. They are selected because further division of the mesh size and time increment did not significantly increase accuracy.

The welding wire is initially at room temperature and has a hemispherical tip whose temperature coincides with the melting point. As the computation proceeds, the following operations are performed at each time step:

(a) Solving the mass continuity and momentum equations for velocity and pressure.

(b) Using the VOF method to find the location and shape of the free surface.

(c) Calculating the slope, radii of curvature and surface tension of the free surface.

(d) Determining the current density and heat flux distributions on the free surface.

(e) Solving the current continuity equation for voltage and current density.

(f) Solving the energy equation for enthalpy and temperature.

(g) Printing the result and advancing to next step.

When a droplet is detached from the wire tip, its volume and detachment period are recorded. The frequency of metal transfer is then calculated based on the averaged detachment period. The initial few drops, however, are excluded from the calculation since their results are largely influenced by the presumed initial conditions.

\section{Results and discussion}

Simulations have been performed for the welding of mild steel with a $1.6 \mathrm{~mm}$ welding wire, whose material properties are listed in table 1 . The predicted results of metal transfer frequency and geometry of the melting interface have been compared with experiments. Analysis based on the numerical model has been made to study the physics of metal transfer, especially the characteristics of relevant physical variables and the mechanisms of taper formation and globular-spray transition.

Table 1. Material properties of welding wire.

\begin{tabular}{lll}
\hline Density, $\rho$ & $6250 \mathrm{~kg} \mathrm{~m}^{-3}$ & {$[11]$} \\
Kinematic viscosity, $v$ & $2.8 \times 10^{-7} \mathrm{~m}^{2} \mathrm{~s}^{-1}$ & {$[12]$} \\
Melting temperature, $T_{\mathrm{m}}$ & $1723 \mathrm{~K}$ & {$[16]$} \\
Latent heat, $L$ & $2.5 \times 10^{5} \mathrm{~J} \mathrm{~kg}^{-1}$ & {$[16]$} \\
Specific heat of solid, $c_{\mathrm{s}}$ & $700 \mathrm{~J}\left(\mathrm{~kg} \mathrm{~K}^{-1}\right.$ & {$[18]$} \\
Specific heat of liquid, $c_{1}$ & $780 \mathrm{~J}\left(\mathrm{~kg} \mathrm{~K}^{-1}\right.$ & {$[18]$} \\
Surface tension coefficient, $\gamma$ & $1.2 \mathrm{~N} \mathrm{~m}^{-1}$ & {$[11]$} \\
Electrical conductivity, $\sigma$ & $8.54 \times 10^{5}(\Omega \mathrm{m})^{-1}$ & {$[12]$}
\end{tabular}




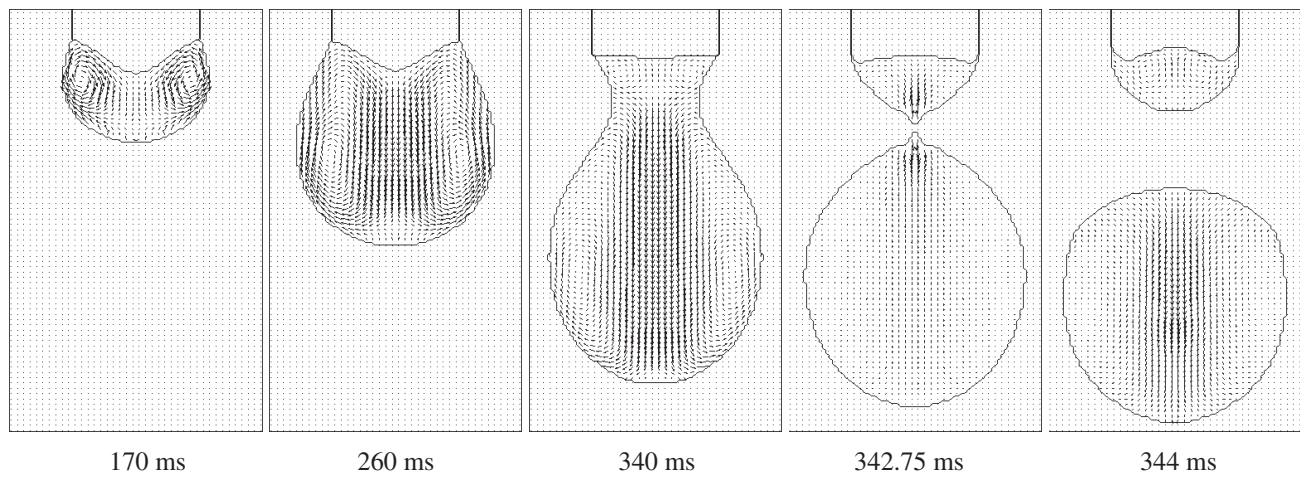

Figure 4. Predicted droplet profile, melting interface, and velocity field in globular transfer mode (1.6 mm wire, $175 \mathrm{~A}, 22 \mathrm{~V}, 95 \mathrm{ipm}$ or $0.0402 \mathrm{~m} \mathrm{~s}^{-1}$, argon).

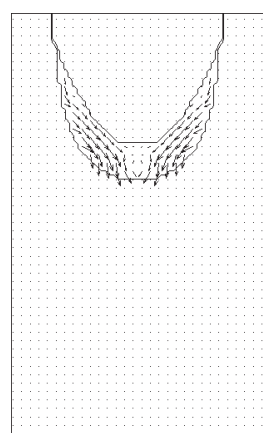

$34.0 \mathrm{~ms}$

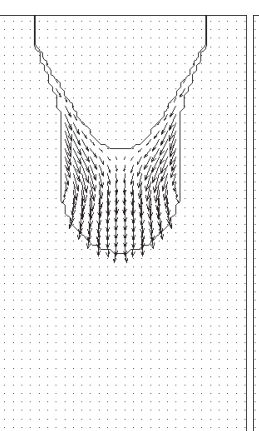

$35.5 \mathrm{~ms}$

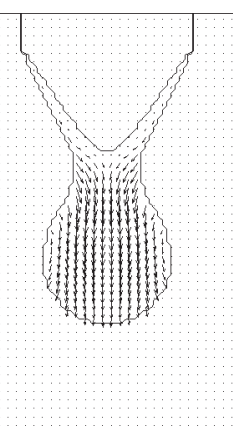

$36.3 \mathrm{~ms}$

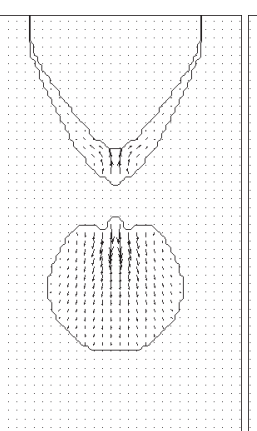

$36.5 \mathrm{~ms}$

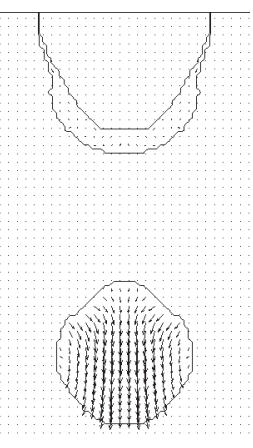

$37.0 \mathrm{~ms}$

Figure 5. Predicted droplet profile, melting interface, and velocity field in spray transfer mode $(1.6 \mathrm{~mm}$ wire, $350 \mathrm{~A}, 30.5 \mathrm{~V}, 218 \mathrm{ipm}$ or $0.0925 \mathrm{~m} \mathrm{~s}^{-1}$, argon).

\subsection{Dynamic variations of droplet profile, melting interface and fluid flow}

Figures 4 and 5 show the simulated droplet profiles, melting interfaces and velocity fields in globular transfer and spray transfer, respectively. For the purpose of illustration, only one transfer period is displayed in each figure, and irregular time intervals are used. In figure 4 , the current is $175 \mathrm{~A}$ and the predicted transfer frequency is 5 drops per second; in figure 5, the current is $350 \mathrm{~A}$ and the predicted transfer frequency is 320 drops per second.

Figures 4 and 5 also show that the melting interface on the welding wire is not flat and it interacts with the liquid flow in the molten droplet. The shape of the interface influences the liquid flow in the droplet through the skin friction along the curve. The liquid flow, on the other hand, affects the shape of the interface through heat convection. Since the welding wire is melted by resistive heating and anode reactions, and, to some extent, also by arc heating, the wire surface directly exposed to the arc tends to melt faster than the interior metal. Therefore, the melting interface usually has a convex shape. However, near the final stage of the droplet detachment, a local depression at the centre of the melting interface may be generated due to an upward recoil flow caused by the unbalanced surface tension. When it occurs, the melting interface becomes crater-shaped as shown in the last plot of figure 4 . This crater-shaped interface lasts a few milliseconds in each transfer period.

Metallographic experiments have been conducted to verify the shape of the melting interface. Figure 6 shows the

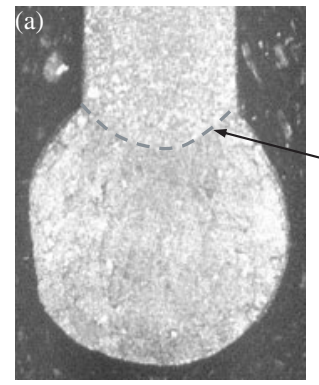

Droplet in globular transfer

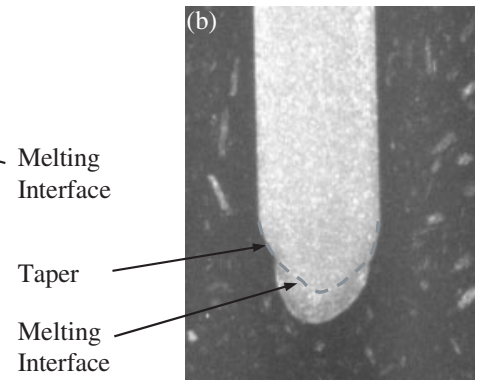

Droplet in spray transfer
Figure 6. Metallographic images of the curved melting interface at the wire tip.

typical cross-sectional images under a microscope. Like the cross-section of a weld bead, the ever-melted but solidified metal has a different microstructure from the unmelted metal. The images prove that the melting interface was not flat in general but convex in most cases.

When a droplet is detached, the heavily deformed surface generates a large surface tension on the liquid that remains at the wire tip. The unbalanced surface tension causes the liquid to recoil and oscillate. Figure 7 shows the evolution of the surface profile and the velocity field due to the recoiling and oscillation.

As the remaining liquid oscillates, the detached droplet also changes in shape, as shown in figure 8 . Immediately after the detachment, the rebound of the liquid near the breakup point generates a small indentation on the top surface. As 


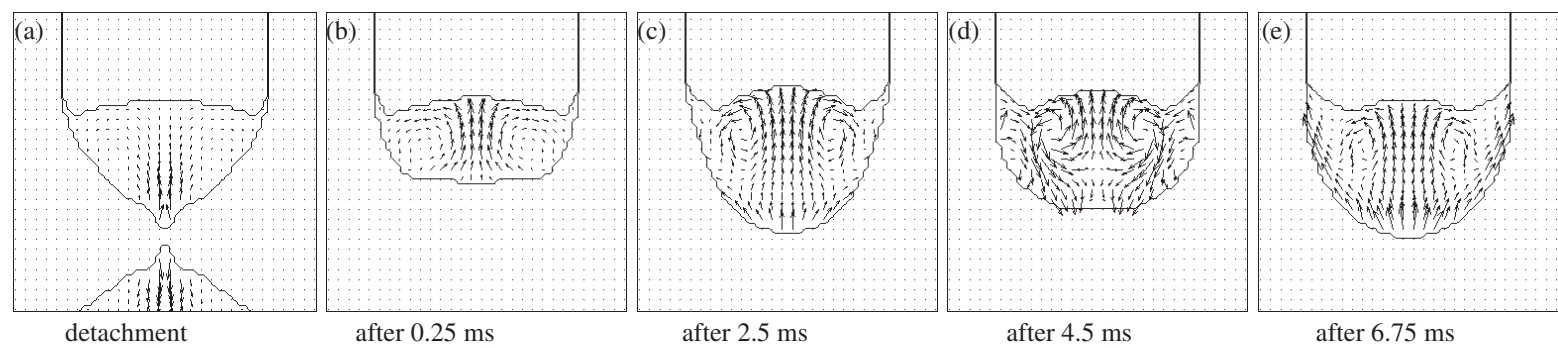

Figure 7. Fluid oscillation at the wire tip after a droplet is detached (175 A).

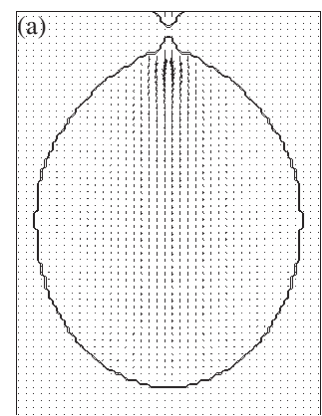

detachment (b)

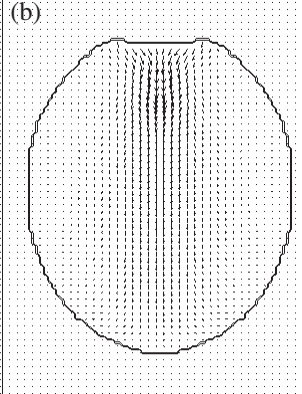

after $0.25 \mathrm{~ms}$ (c)

c) (d) (e)

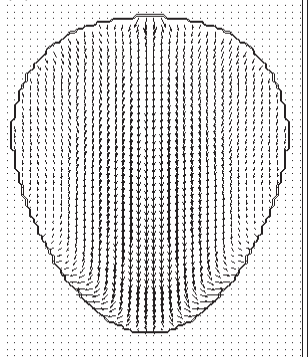

after $5.25 \mathrm{~ms}$

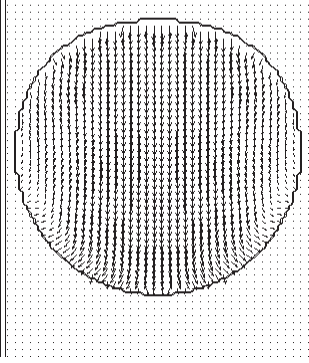

after $9.25 \mathrm{~ms}$

Figure 8. Shape of droplet after detachment (175 A).

the indentation quickly recovers, the droplet also deforms and oscillates in the axial direction. The oscillation dies out in a few milliseconds due to the viscous damping. After that, the droplet continues to move to the weld pool with a relatively constant shape.

\subsection{Characteristics of physical variables and their roles in metal transfer}

Metal transfer is influenced by a number of physical variables, including temperature, velocity, current density, electric potential, magnetic field, electromagnetic force, and pressure. The characteristics of these variables at two representative stages (droplet growth and pre-detachment) are analysed in this subsection.

Figure 9 shows the distributions of these variables when the droplet is growing under $175 \mathrm{~A}$. In the temperature plot, $\mathrm{V}$-shaped or U-shaped isothermal contours are predicted. The highest temperature is located at the bottom and near the surface of the droplet. This is caused by the concentrated heating on the anode spot and the convection pattern within the droplet. Under the continuous arc heating, the temperature of the droplet surface will reach the boiling point and the metal will start to vaporize. Since the cooling effect of metal vaporization is ignored in the present model, the predicted temperature near the droplet surface may be higher than the boiling point. This suggests that the metal vaporization deserves further consideration in the future development of a more accurate metal transfer model. In the velocity plot, the liquid near the centreline of the droplet moves downward from the droplet root and circulates upstream along the free surface. In the current density plot, most of the current flows downward and parallel to the symmetry axis, but slight divergence exists near the droplet surface. The electric potential, or voltage, exhibits a nearly one-dimensional distribution. Since the main component of the current is in the axial direction, the voltage also drops in this direction. In the plot of magnetic field, the centre of the droplet has a low value and the surface, especially the area near the root of the droplet, has a high value. Similar to the magnetic field, the electromagnetic force, or Lorentz force, also reaches its maximum value at the surface near the droplet root, and has a relatively low value in the centre of the droplet. This explains why necking occurs near the root of the droplet. In the pressure plot, the local pressure near the surface depends on the curvature of the surface. Larger curvature induces greater pressure.

Figure 10 shows the distributions of these variables when detachment is about to occur. In the temperature plot, the highest temperature is still located at the bottom and near the surface of the droplet. The temperature in the centre of the droplet 'neck' is moderate. In the velocity plot, the liquid below the neck has high velocity away from the wire toward the workpiece, and the liquid above the neck circulates upstream near the symmetry axis and downstream near the droplet surface. The centre of the neck is a velocity stagnation point. In the horizontal neighbourhood of this point, the liquid moves towards the centre and then goes either downward or upward. In the plot of current density, the current at the wire tip first converges towards the droplet neck and then diverges after passing through it. The current density in the centre of the neck is more than 50 times of that in the wire. Due to the highly concentrated current density and greatly increased electrical resistance, the voltage drop on the neck is very large. Although physically connected, the voltage difference between the solidliquid interface and the centre of the droplet can be as high as several volts at the moment before breakup. The high current density at the neck also generates a high magnetic field and a large electromagnetic force. The maximum value of the 


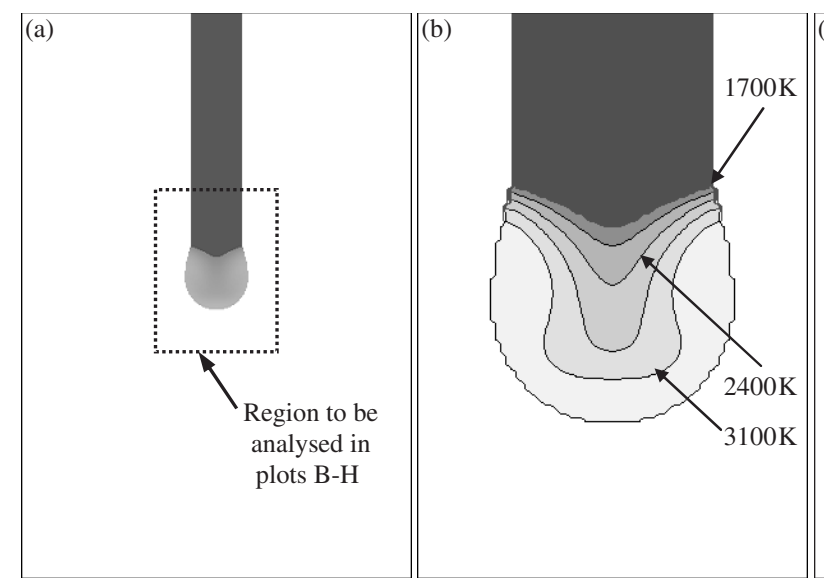

Complete Domain

Temperature

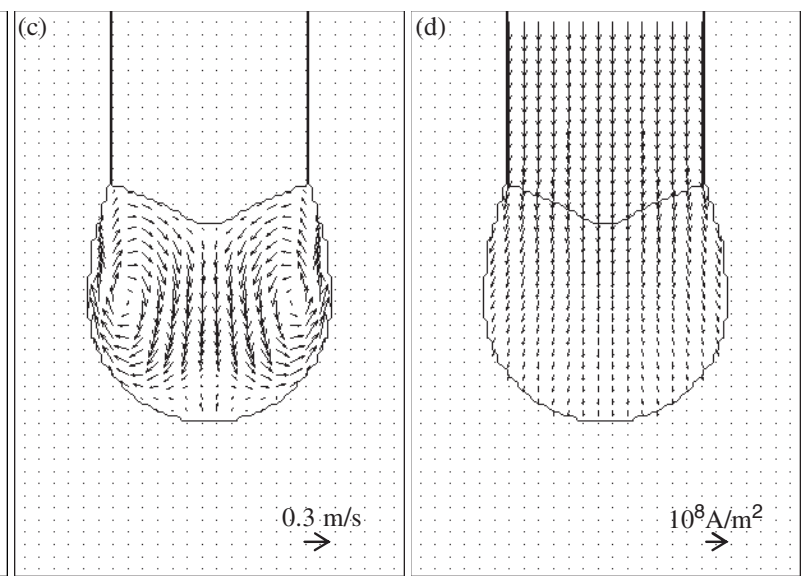

Velocity Relative to WFS

Current Density
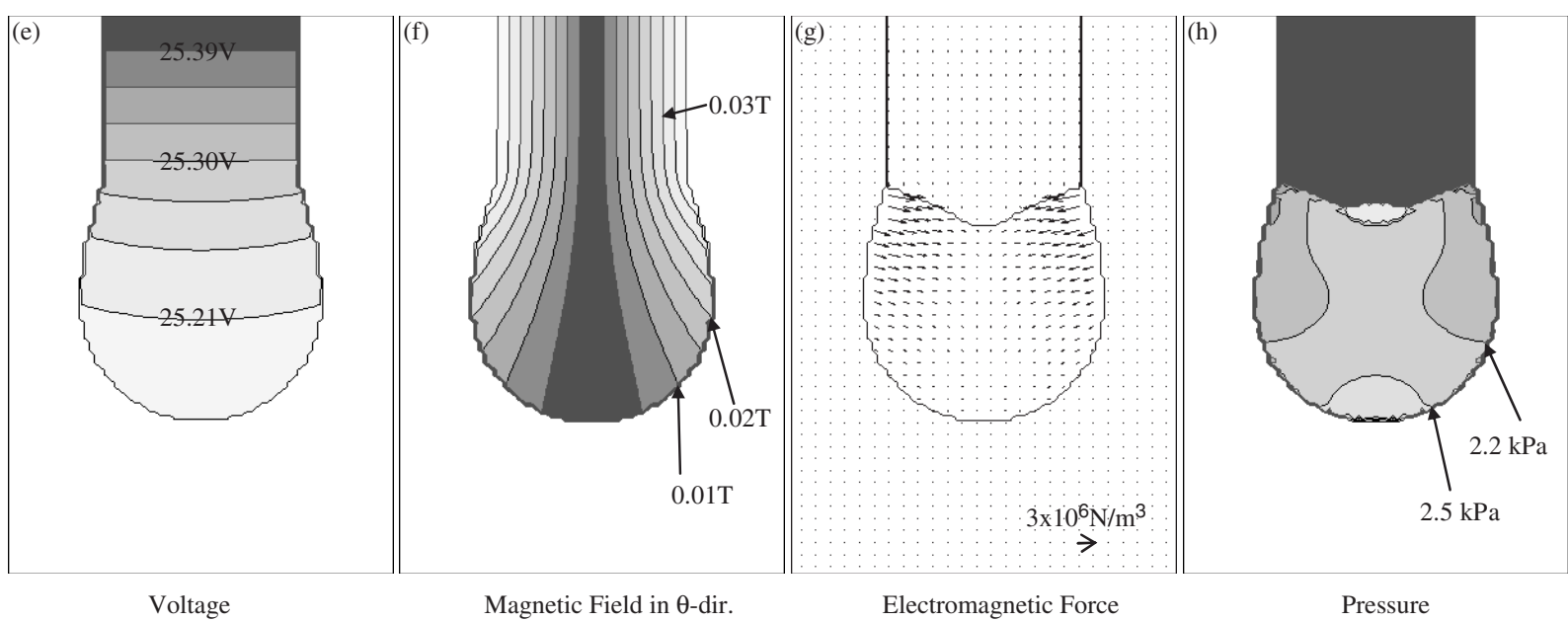

Figure 9. Distribution of physical variables during the droplet growth (175 A).

electromagnetic force is more than $10^{4}$ times that of gravity. As a result of the large electromagnetic force and the surface tension due to the local curvature, the pressure reaches a peak value in the centre of the droplet neck. It causes the liquid to be squeezed out of the neck and thus accelerates the detachment process.

\subsection{Mechanisms of taper formation and globular-spray transition}

The transition between globular transfer and spray transfer is an important subject in GMAW. However, there are two differential observations of this phenomenon. The long accepted observation was reported in 1958 by Lesnewich [28], who described the transition as a sharp change in a very narrow current range of $10 \mathrm{~A}$, as shown by the thin dashed line in figure 11. But in 1993, Kim and Eagar [7] found that the transition occurred 'much more gradually than was generally believed'. Their results are reproduced in figure 11 as black dots. As different descriptions of the phenomenon exist, there are also different explanations of the mechanism that causes the globular-spray transition. The major difference of these explanations is about the role of taper formation in globularspray transition. Allum [5] considered the taper formation as an important factor in the globular-spray transition as it reduces the effective fluid diameter and accelerates the droplet detachment. However, Choi et al [12], predicted the globular-spray transition without explicitly modelling the reduction of wire diameter caused by the taper. In other words, they believed that the increased electromagnetic force due to higher current was the cause, and both taper formation and globular-spray transition were the results.

We have conducted experiments to observe the metal transfer using a high-speed motion analyser and the lasershadow imaging method [29]. The frequency of metal transfer is calculated by counting the number of detached droplets in a unit time, and the result is illustrated in figure 11 as a thick dashed line. Comparison between this curve and the two curves mentioned earlier shows that our observation is closer to Kim and Eagar's result, that is, the globular-spray transition is a gradual rather than a sudden process. As will be shown later, the conclusion of gradual globular-spray transition is supported by simulations.

Also appearing in figure 11 are three simulation curves, each featuring a different value of $\Delta z$, indicating the extent of heat flux coverage and thus the amount of heat input on the unmelted wire (see figure 3). When the current is below $250 \mathrm{~A}$ (globular transfer), the predicted metal transfer frequency is independent of $\Delta z$. But when the current is higher than $250 \mathrm{~A}, \Delta z=0$ gives higher transfer frequencies than the 


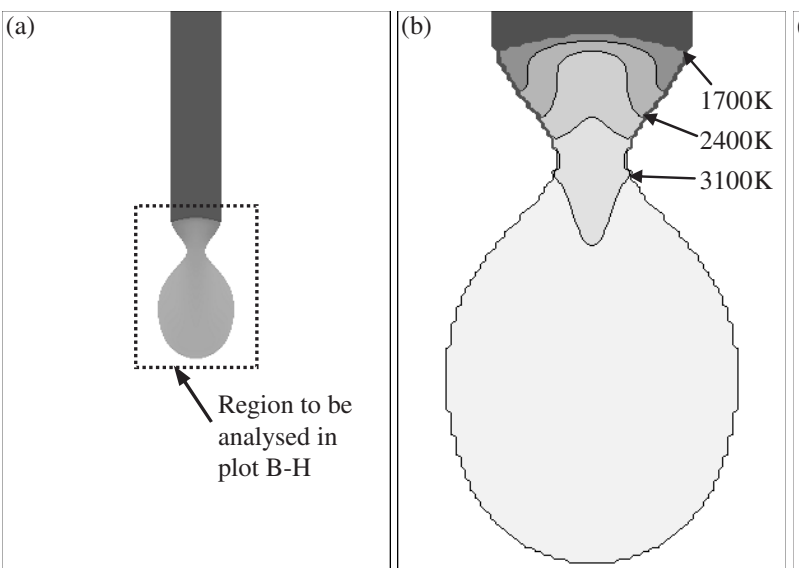

Droplet Profile

Temperature

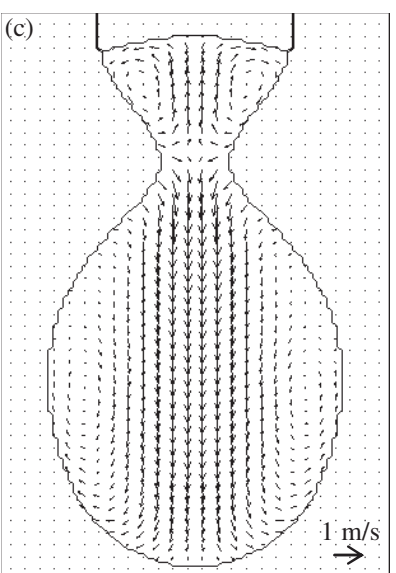

Velocity relative to WFS

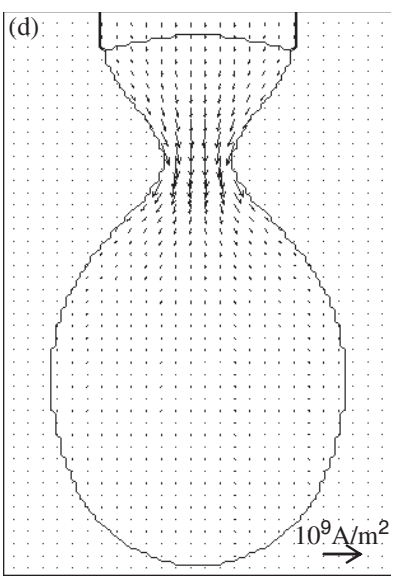

Current Density (e)

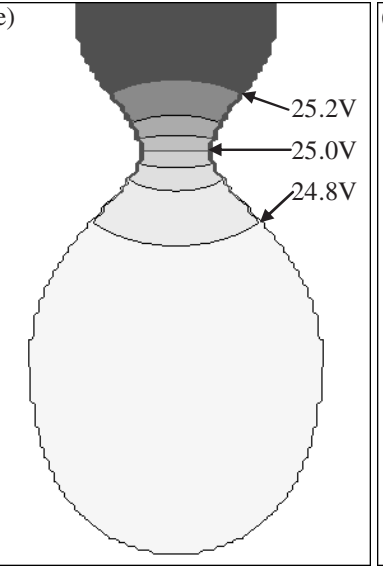

Voltage

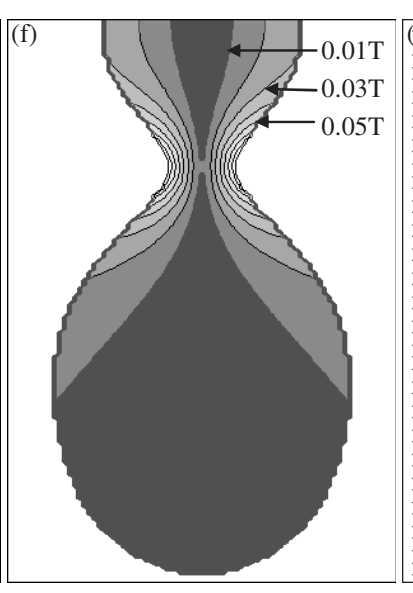

Magnetic Field in $\theta$-dir.

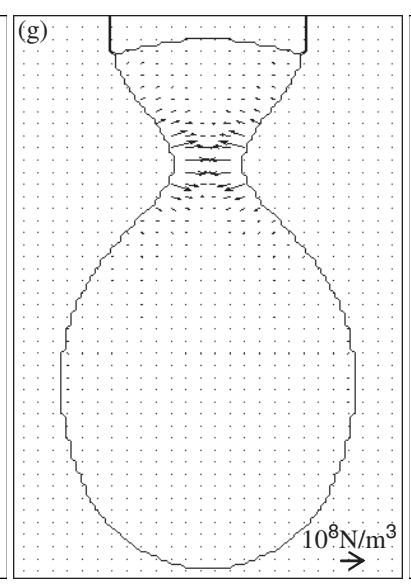

Electromagnetic Force

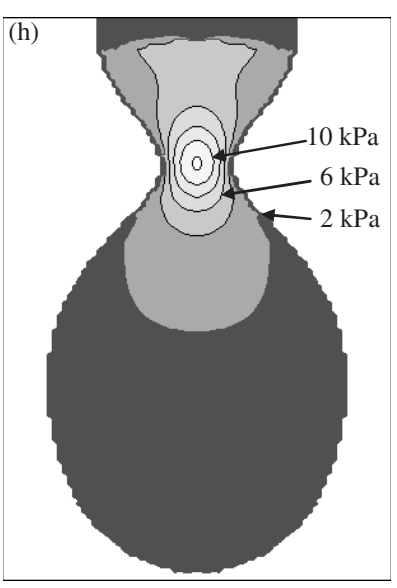

Pressure

Figure 10. Distributions of physical variables before droplet detachment (175 A).

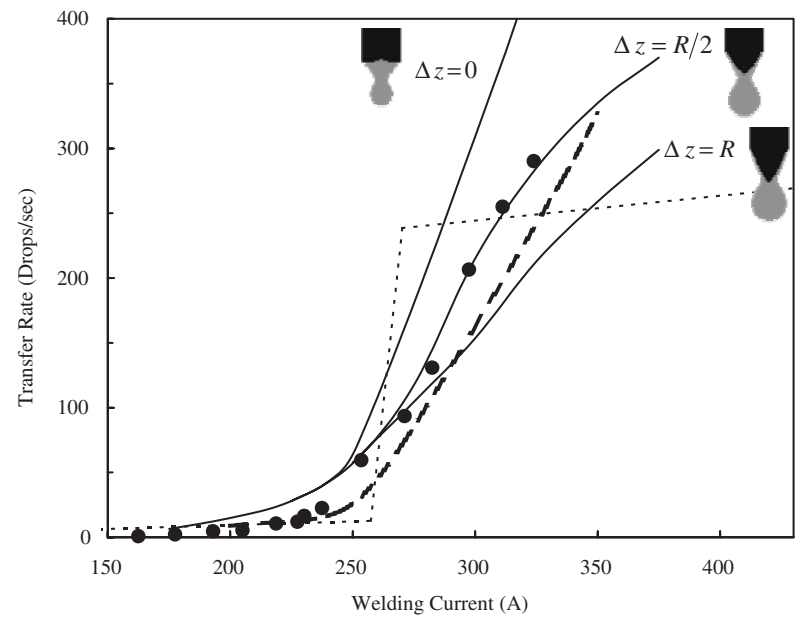

- - Experiment (Wang et al) $\quad \cdots \cdot$ - - - Lesnewich [26]

- Kim and Eagar [7] — - _ Simulations (Wang et al)

Figure 11. Comparison of simulation and experimental results.

measured values, and $\Delta z=R$ gives lower frequencies than the measured values, where $R$ is the radius of the welding wire. When $\Delta z=R / 2$, the predicted frequency curve agrees very well with our experiments and the experiments conducted by
Kim and Eagar. Hence, we use $\Delta z=R / 2$ in most of our calculations in this paper.

Along with the three simulation curves are also the images of the simulated droplet profiles and wire tip geometries. Experimental observation shows that, when the current is high, a taper is formed at the tip of the wire. But if we simulate the process with $\Delta z=0$, or if we completely ignore the heat input on the unmelted wire, no taper can be predicted. In contrast, if we use $\Delta z=R / 2$ or $\Delta z=R$, the taper formation is simulated. This means that the thermal balance on the unmelted welding wire plays an important role in taper formation. This can be seen from figure 12 , showing the wire tip geometry as well as the distributions of temperature, velocity and electromagnetic force in the droplet when a taper is formed. The heat input on the unmelted portion of the wire preheats the wire surface to an elevated temperature such that the wire surface tends to melt faster than the interior metal. When the current increases, the height of the convex melting interface also increases. The increased electromagnetic force pinches the molten fluid and drives it move along the sloped surface to the bottom of the wire tip. Because of the liquid flow along the sloped surface, a thin liquid layer is formed on the surface. This thin liquid layer allows the arc heat to penetrate such that the melting proceeds in the direction perpendicular to the sloped wire surface. Figure 12 also shows that the taper formation is 


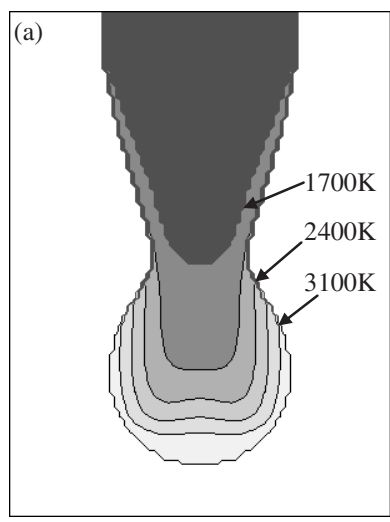

Temperature

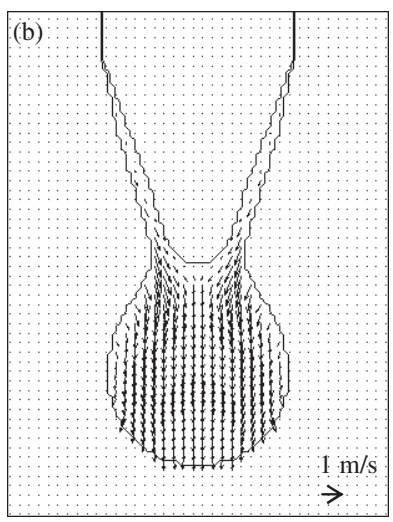

Velocity relative to WFS

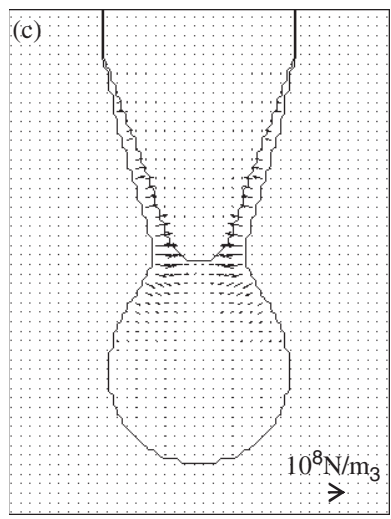

Electromagnetic force

Figure 12. Taper formation at wire tip in spray transfer ( $350 \mathrm{~A})$. A thin layer of molten liquid moves along the sloped melting interface.

different from the necking of a liquid column. For the necking of a liquid column, the diameter of the liquid shrinks due to external forces. But for taper formation, a solid wire first becomes tapered and the liquid moves along the tapered core and reduces in external diameter.

Although no taper is predicted in figure 11 when $\Delta z=0$, the frequency curve corresponding to $\Delta z=0$ exhibits a quick increase when the current is higher than $250 \mathrm{~A}$. This means the globular-spray transition is not caused by the taper formation, but by the increased electromagnetic force. However, figure 11 shows that the taper formation affects the globular-spray transition by altering the slope of the frequency curve in the transition range. We find that the existence of a taper does not accelerate the metal transfer, but rather decelerates the process and reduces the transfer frequency. When no taper is formed, most of the current passes through the droplet neck and generates an extremely large electromagnetic force at the neck. The necking location is close to the cross-section that transmits the maximum current. But when a long taper is formed, a fraction of the total current leaves the droplet surface before it reaches the necking location, and therefore the electromagnetic force at the neck reduces and the detachment process is decelerated.

\subsection{Generation of satellite drops}

When a droplet is detached, the thin liquid bridge connecting the droplet and the wire tip may also break and evolve to one or more tiny droplets. These droplets are known as satellite drops. In GMAW, the volume of a satellite drop is only about $1 \%$ of that of the primary drop, but it can be observed by using a high-speed camera. Figure 13 shows an example of the satellite formation [30]. With finer mesh, the satellite formation can also be simulated by using the numerical model developed in this paper, as shown in figure 14 .

Satellite drop generation is not a desirable phenomenon, since the small droplets may end up as spatter and stick to the workpiece or gas nozzle. Controlling of the satellite formation requires further understanding of its mechanism. Empirical formulae based on nondimensional numbers have been derived to predict the satellite formation in water drops [31]. While similar procedures can be taken to derive empirical equations for GMAW, numerical simulation can also be employed to predict the satellite formation.

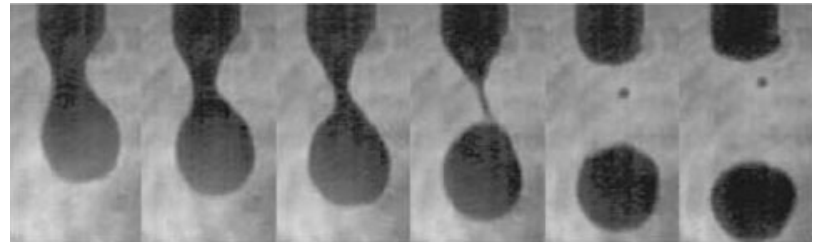

Figure 13. Formation of a satellite drop (source: Jones et al 1998 [30]).
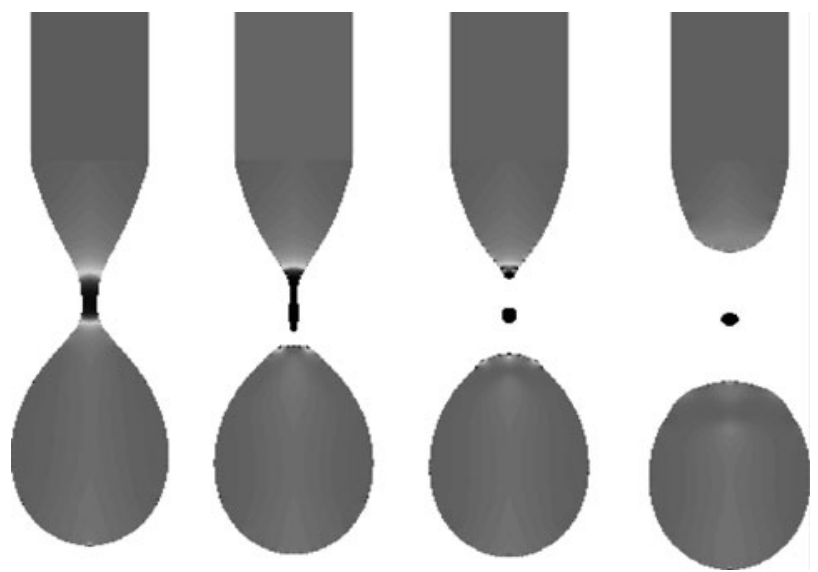

Figure 14. Simulation of satellite formation.

\section{Conclusions}

(1) A nonisothermal numerical model is developed to simulate the metal transfer process in GMAW. Experiments with high-speed photography, laser-shadow imaging and metallographic analysis show that the simulation results are in broad agreement with the actual welding process. The model can also be readily extended to the weld pool so that a unified simulation system can be developed to describe the complete GMAW process.

(2) Using the numerical model to study the physics of metal transfer, we find that the taper formation at higher welding current is closely related to the heat input on the unmelted portion of the welding wire; the globularspray transition is mainly due to the increased current and electromagnetic pinch force, and the taper formation 
influences this transition by decelerating the transfer process; the geometry of the melting interface on the welding wire experiences dynamic variations during a metal transfer period, and it interacts with the liquid flow in the droplet; before a droplet is detached, the centre of the droplet neck experiences an extremely large current density, magnetic field, electromagnetic force and hydrodynamic pressure; after a droplet is detached, the unbalanced surface tension causes the remaining liquid to recoil and oscillate on the wire tip; and satellite drops may be generated following the detachment of the primary droplets.

(3) Future improvement of the model can be made by integrating the following effects: (a) metal vaporization on the droplet surface; (b) a plasma model that computes rather than imposes electrical and thermal fluxes; and (c) Marangoni and drag effects on droplet surface.

\section{Acknowledgments}

This research is supported by General Motors through the GM Collaborative Research Laboratory at the University of Michigan. The authors would like to express their sincere thanks to Dr Samuel P Marin from GM R\&D for his support and comments.

\section{References}

[1] Lancaster J F 1986 The Physics of Welding 2nd edn (New York: Pergamon)

[2] Greene W J 1960 An analysis of transfer in gas-shielded welding arcs Trans. AIEE Part II 79 194-203

[3] Amson J C 1962 An analysis of the gas-shielded consumable metal arc welding system $B r$. Weld. J. 41 232-49

[4] Allum C J 1985 Metal transfer in arc welding as a varicose instability: I. Varicose instability in a current-carrying liquid cylinder with surface charge J. Phys. D: Appl. Phys. 18 1431-46

[5] Allum C J 1985 Metal transfer in arc welding as a varicose instability: II. Development of model for arc welding J. Phys. D: Appl. Phys. 18 1447-68

[6] Maruo H, Hirata Y and Goto N 1992 Bridging transfer phenomena of conductive pendent drop-the effects of electromagnetic pinch force on the bridging transfer Quart. J. Japan Weld. Soc. $1043-50$

[7] Kim Y S and Eagar T W 1993 Analysis of metal transfer in gas metal arc welding Weld. J. 72 269-s-78-s

[8] Nemchinsky V A 1994 Size and shape of the liquid droplet at the molten tip of an arc electrode J. Phys. D: Appl. Phys. 27 1433-42

[9] Simpson S W and Zhu P 1995 Formation of molten droplets at a consumable anode in an electric welding arc J. Phys. D: Appl. Phys. 28 1594-600

[10] Hirata Y, Osamura T, Goto N and Ohji T 1997 Numerical model of short-circuiting transfer process in GMA welding Proc. 7th Int. Conf. on Computer Technology in Welding (San Francisco, California, 8-11 July 1997)
[11] Jones L A, Eagar T W and Lang J H 1998 A dynamic model of drops detaching from a gas metal arc welding electrode J. Phys. D: Appl. Phys. 31 107-23

[12] Choi B K, Yoo C D and Kim Y S 1998 Dynamics simulation of metal transfer in GMAW-Part 1: globular and spray transfer modes Weld. J. 77 38-s-44-s

[13] Choi B K, Ko S H, Yoo C D and Kim Y S 1998 Dynamic simulation of metal transfer in GMAW-Part 2: short-circuit transfer mode Weld. J. 77 45-s-51-s

[14] Zhang Y M and Liguo E 2000 Numerical analysis of the dynamic growth of droplets in gas metal arc welding Proc. Inst. Mech. Eng., Part C: J. Mech. Eng. Sci. 214 1247-58

[15] Haidar J and Lowke J J 1996 Predictions of metal droplet formation in arc welding J. Phys. D: Appl. Phys. 29 2951-60

[16] Fan H G and Kovacevic R 1998 Dynamic analysis of globular metal transfer in gas metal arc welding - a comparison of numerical and experimental results J. Phys. D: Appl. Phys. 31 2929-41

[17] Fan H G and Kovacevic R 1999 Droplet formation, detachment, and impingement on the molten pool in gas metal arc welding Metall. Mater. Trans. B-Process Metall. Mater. Process. Sci. 30 791-801

[18] Wang Y and Tsai H L 2001 Impingement of filler droplets and weld pool dynamics during gas metal arc welding process Int. J. Heat and Mass Transfer 44 2067-80

[19] Kou S and Sun D K 1985 Fluid flow and weld penetration in stationary arc welds Metall. Trans. A 16 203-13

[20] Hirt C W and Nichols B D 1981 Volume of fluid (VOF) method for the dynamics of free boundaries J. Comput. Phys. 39 201-25

[21] Cobine J D and Burger E E 1955 Analysis of electrode phenomena in the high-current arc J. Appl. Phys. 26 895-900

[22] Nemchinsky V A 1997 Heat transfer in a liquid droplet hanging at the tip of an electrode during arc welding J. Phys. D: Appl. Phys. 30 1120-4

[23] Nemchinsky V A 1997 Electrode evaporation in an arc with pulsing current J. Phys. D: Appl. Phys. 30 2895-9

[24] Kim Y S and Eagar T 1989 Temperature distribution and energy balance in the electrode during GMAW Recent Trends in Welding Science and Technology: Proc. 2nd International Conference on Trends in Welding Research (Gatlinburg, Tennessee, 14-18 May 1989) ed S A David and J M Vitek (Ohio: Materials Park) pp 13-18

[25] Jonsson P G, Eagar T W and Szekely J 1995 Heat and metal transfer in gas metal arc welding using argon and helium Metall. Mater. Trans. B 26 383-95

[26] Zhu P, Rados M and Simpson S W 1997 Theoretical predictions of the start-up phase in GMA welding Weld. $J$. 76 269-s-74-s

[27] Grong O 1997 Metallurgical Modeling of Welding (London: The Institute of Materials)

[28] Lesnewich A 1958 Control of melting rate and metal transfer in gas-shielded metal arc welding: part II-control of metal transfer Weld. J. 37 418-s-25-s

[29] Rhee S and Kannatey-Asibu E 1992 Observation of metal transfer during gas metal arc welding Weld. J. 72 381-s-5-s

[30] Jones L A, Eagar T W and Lang J H 1998 Magnetic forces acting on molten drops in gas metal arc welding J. Phys. D: Appl. Phys. 31 93-106

[31] Zhang X 1999 Dynamics of growth and breakup of viscous pendant drops into air J. Colloid Interface Sci. 212 107-22 